\title{
The Shared Decision for Osteoarthritis Treatments
}

ISSN: 2576-8875

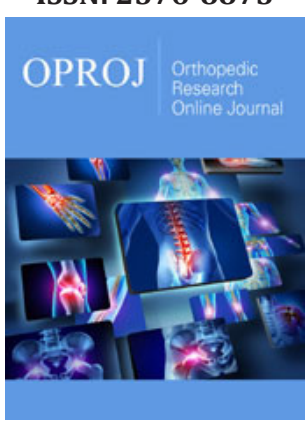

${ }^{* 1}$ Corresponding author: Basem Al-Omari, Faculty of Health and Life Sciences, Coach Lane Campus East, Room H217, Northumbria University, Newcastle-upon-Tyne, NE7 7XA

Submission: 留 July 19, 2019

Published: 䒸 July 30, 2019

Volume 5 - Issue 5

How to cite this article: Al-Omari $\mathrm{B}$, Bate A, McMeekin P. The Shared Decision for Osteoarthritis Treatments. Ortho Res Online J. 5(5). OPROJ.000625.2019. DOI: 10.31031/OPROJ.2019.05.000625

Copyright@: Basem Al-Omari, This article is distributed under the terms of the Creative Commons Attribution 4.0 International License, which permits unrestricted use and redistribution provided that the original author and source are credited.

\author{
Basem Al-Omari*, Angela Bate and Peter McMeekin
}

Faculty of Health and Life Science, Northumbria University, UK

\begin{abstract}
In recent years, there has been increasing interest in collaborative, shared decision-making between physicians and patients. The shared decision-making process involves healthcare professionals and patients working together to make a healthcare decision. For patients and clinicians to reach mutual decisions about treatment, they must have an understanding of the condition, treatment options available, and the risks and benefits of each treatment. This is a very complex process when dealing with osteoarthritis (OA) patients.
\end{abstract}

Treatment recommendations for OA vary depending on several factors, which results in patients having to continuously trade-off these factors against each other. Another challenge is that these factors differ between individuals and healthcare settings. Therefore, healthcare professionals must understand patients' preferences to achieve a shared decision and be able to reach a mutual agreement regarding $\mathrm{OA}$ treatment. This is a complicated process that requires time, effort, and expertise.

\section{Introduction}

\section{Osteoarthritis patients' treatment preferences}

Generally, pharmacological treatment guidelines are based on extensive clinical data, e.g. Randomised Controlled Trials (RCTs). Although research evidence such as RCTs regarding the effectiveness of treatment is one of the fundamental components of evidence-based practice, alone it is insufficient for making decisions about patient care [1]. Healthcare practitioners are encouraged to implement evidence-based practice in conjunction with patient-centred care [2]. Recently, there has been increased attention toward combining patient centred care and evidence-based practice despite tension and conflict between the two [3]. Since then, many researchers and academics focused on the inclusion of patients' preferences and values in the decision-making process.

In the UK, the NICE OA guidelines [4] suggest that treatment and care of OA should take into account patients' treatment preferences and beliefs, especially when there is uncertainty about the probability of treatment outcomes, or when there are two or more treatment options that offer a similar balance between benefits and risks [5]. Taking into account patients' preferences is advocated as an optimal model of collaborative care between patients and clinicians [5]. Patients' preference is also considered to influence patients' compliance, which is one of the vital components of the successful management of OA pain [6]. Patients' values and preferences contribute to patients' concordance of any prescribed treatment. Simply, if the patients did not like the treatment, they are not going to adhere to it. Therefore, patients' values and preferences, should be considered alongside clinicians' expertise and best available evidence. Prioritising treatments most important to the patient requires considering the individual patient's preferences and trade-offs as well as the expression of their particular pattern of illness and the significance of particular symptoms [7], therefore, Clinicians face substantial challenges in dealing well with chronic illnesses [8].

In $\mathrm{OA}$, treatment recommendations vary depending on symptom severity and patients' prior experience with other treatments. It is also suggested that if health care practitioners know more about patients' preferences, care could be cheaper and more effective [6]. In a cross-sectional study assessing the relation between patients' beliefs and reported adherence [9], it was found that over a third of the patients had strong concerns about their 
medication based on beliefs about the dangers of dependence or long-term effects. These results suggest that patients' beliefs about the need for medication are weighed against concerns about the potential adverse effects. Thus, patients' medication preferences may influence their compliance with treatment. As a predictor of patients' compliance, patient beliefs about the medication were found to be more powerful than clinical and sociodemographic factors. Indeed, this accounted for $19 \%$ of the explained variance in compliance [9].

For patients to make informed decisions about their care, they need to understand their treatment options [10]. When patients are making these decisions about their preferred treatment choice, they are considering different characteristics of this treatment such as the method of administration, effectiveness, side effects, etc [11]. Furthermore, patient preferences about medication type for chronic knee pain is affected by many factors, e.g. previous experience of medication, other illness, pain elsewhere, convenience and severity of pain [12]. The pharmacological treatment recommended for mild OA symptoms (paracetamol) has modest efficacy but no inherent risks of adverse effects, while potentially more effective options, such as NSAIDs or opioids, present a higher risk of side effects $[5,13]$. Therefore, patients with OA are continually faced with decisions that imply relevant trade-offs between benefits and risks [5]. However, we do not currently have a clear picture of the relative importance of the different factors influencing OA patients' medication preferences or if these factors differ individually or according to the healthcare settings [7]. Identifying the relative importance of these factors may allow physicians to prescribe medications that match patients' preferences. For example, it would be easier for the physicians to prescribe medication to a patient who has mild $\mathrm{OA}$ if the physicians were aware that the patient had a preference for avoiding side effects over the method of administration and frequency of use.

A study conducted by Landmark and colleagues [14] exploring how physicians elicit patient preferences in shared decision-making and found that physicians occasionally present a treatment option to the patients based on hypothetical patients' preferences that are made by physicians themselves which constrains the patient's freedom of choice and exerts a pressure to accept the physician's recommendation. In other occasions, physicians assume that older patients with limited health knowledge may not want to participate in the treatment decision making process [15]. These assumptions are inconsistent with both the evidence and contemporary models of care [16]. Patients' preferences are vital when considering multi attributes treatment options for patients [17]. Matching patients' preferences to treatments has been shown to be effective in symptom reduction [18]. Thus, treatment decisions are dependent on patient preferences [14]. However, it must be acknowledged that eliciting patients' preferences is a complicated process that requires time, effort, and expertise.

The United Kingdom (UK) government's vision of the National Health Services (NHS) in England is for patients and clinicians to reach mutual decisions about treatment, with a shared understanding of the condition, the options available, and the risks and benefits of each of those [19] which resulted in the government producing a proposal for "no decision about me, without me" policy. This document included proposals to give the patients and public more say over their care and treatment with more opportunity to make informed choices, as a means of securing better care and better outcomes [20]. The main emphasis of the policy was to create a culture where the shared decision making is the standard procedure during the clinician-patient consultation.

\section{Shared decisions and eliciting patients' preferences re- garding osteoarthritis treatments}

The process that involves health professionals and patients working together to make healthcare decision is called "shared decision making" [21]. Shared decision making is particularly recommended for preference-sensitive medical decisions, where two or more equivalent treatment options are available or consequences of the treatment affect the patients' daily life [18]. Therefore, a shared decision-making approach is suitable in the treatment of chronic conditions [22] such as OA.

Shared decision-making can be considered as "a bilateral process that leads to a joint and equivalent treatment decision based on shared information between clinician and patient" [23]. This highlights the importance of both the information provided to patients and the information received from them regarding their preferences. Carnes [24] found that shared decisions, where doctors take account of patient preferences, encourage compliance with the treatment process and positive perception of the drug used. To facilitate this process of shared decision making between clinicians and their $\mathrm{OA}$ patients, clinicians must understand patients' preferences for $\mathrm{OA}$ treatment, which requires the understanding of factors such as patients' perceptions and beliefs associated with the different treatment options [25]. Clinicians could use the information of the trade-off that OA patients make, to tailor treatment to match the patients' preferences [26].

The King's Fund [19] suggests that the principle of shared decision-making in the context of a clinical consultation should:

a) support patients to articulate their understanding of their condition and of what they hope treatment (or self-management support) will achieve

b) inform patients about their condition, about the treatment or support options available, and about the benefits and risks of each

c) ensure that patients and clinicians arrive at a decision based on mutual understanding of this information

d) record and implement the decision reached.

These points were some of the main principles of the "no decision about me, without me" policy. However, in order for this process to be successful, clinicians must understand patients' preferences, beliefs, and knowledge about the treatment.

The process of eliciting patients' preferences can be complicated and time-consuming. Thus, the expectation that patients become 
more involved in making treatment decisions poses a challenge for doctors [27]. This challenge could be due to several reasons such as; that patients vary in how much they would like to be involved in the treatment decision-making process, their treatment preferences may change over time [28], and treatment options may include several factors to be considered [29]. Therefore, methods of eliciting patients' preferences are essential tools for practitioners to involve their patients in the shared-decision making process. Frewer [25] suggest that to take full account of what patients consider as best treatment, there is a need to develop an innovative method that is sensitive to the differences in patient preferences. Therefore, work is needed with patients to gain their perspectives to understand how a method of eliciting preference for use in routine primary care might be structured [8]. This suggests that patients must not only be involved in the consultation itself, but they must also be consulted and involved in the development of a practical method to elite their preferences [17].

There are many methods of studying patients' preferences concerning OA treatment [30]. Qualitative methods have been used to study patients' decisions regarding OA medication and some, but a limited number of the previous work have used standard quantitative questionnaires to determine patients' preferences [26].

There is a suggestion that the trade-off that OA patients make of one treatment attribute against another has not been widely studied [31], which highlights the need for methods to understand this trade-off. The need for such methods encouraged behavioural scientists to develop alternative techniques to traditional survey for eliciting preference that involves studying choice behaviour rather than just asking about the different choices [32]. Bridges and colleagues [33] suggested that the flexibility of the patient preference elicitation method should capture the interest of most outcome researchers.

Conjoint analysis is one method that can be used to study preferences [34], by quantifying the relative influence of different treatment factors on decision-making [35]. The most advanced conjoint analysis technique is Adaptive Choice Based Conjoint (ACBC) that used and comprehensively described and evaluated by Al-Omari and colleagues $[7,17,29]$ to elicit patients preferences for OA treatment. ACBC is a computerised and/or web-based interactive conjoint analysis questionnaire that can analyse patients' preferences and has the potential to identify individual preferences as a practical basis for concordant prescribing for $\mathrm{OA}$ in clinical practice $[7,36]$.

\section{Conclusion}

Identifying patients' preferences with respect to OA treatment may allow physicians to prescribe treatments that match patients' preferences, enhance compliance with medications, and enhance patients' satisfaction and coping with this chronic disease. In order to identify patients' preferences regarding the pharmaceutical treatment of $\mathrm{OA}$, there is a need to develop an innovative method that addresses the differences in patient preferences. The ACBC method potentially offers an approach to incorporate patients' preferences into the shared decision-making process regarding $\mathrm{OA}$ treatment.

\section{References}

1. Montori V, Brito J, Hassan M (2013) The optimal practice of evidencebased medicine incorporating patient preferences in practice guidelines. JAMA 310(23): 2503-2504.

2. Siminoff LA (2013) Incorporating patient and family preferences into evidence-based medicine. BMC Med Inform Decis Mak 13(3): 6.

3. Burman M, Robinson B, Hart A (2013) Linking evidence-based nursing practice and patient-centred care through patient preferences. Nursing Administration Quarterly 37(3): 231-241.

4. NICE (2014) Osteoarthritis: care and management. NICE guidline [CG177].

5. Rivero-Santana A, Perestelo-Perez L, Perez-Ramos J, Gonzalez-Lorenzo M, Serrano-Aguilar P (2012) Treatment preferences in patients with knee or hip osteoarthritis: an overview. In osteoarthritis - diagnosis, treatment and surgery, shanghai, intech china, pp. 227-248.

6. Brennan PF, Strombom I (1998) Improving health care by understanding patient preferences: the role of computer technology. JAMIA 5(3): 257262.

7. Al-Omari B, Frisher M, Croft P, Sim J (2017) Generating individual patient preferences for the treatment of osteoarthritis using Adaptive Choice-Based Conjoint (ACBC) Analysis. Rheumatology and Therapy 4(1): 167-182.

8. Mangin D, Stephen G, Bismah V, Risdon C (2016) Making patient values visible in healthcare: a systematic review of tools to assess patient treatment priorities and preferences in the context of multimorbidity. BMJ Open 6: e010903.

9. Horne R, Weinman J (1999) Patients' beliefs about prescribed medicines and their role in adherence to treatment in chronic physical illness. Journal of Psychosomatic Research 47(6): 555-567.

10. Kinsey K, Firth J, Elwyn G, Edwards A, Brain K, et al. (2017) Patients' views on the use of an option Grid for knee osteoarthritis in physiotherapy clinical encounters: An interview study. Health Expect 20(6): 13021310.

11. Fraenkel L, Bogardus ST, Concato J, Wittink DR (2004) Treatment options in knee osteoarthritis: the patient's perspective. Archives of Internal Medicine 164(12): 1299-1304.

12. Underwood M, Ashby D, Carnes D, Castelnuovo E, Cross P (2008) Topical or oral ibuprofen for chronic knee pain in older people. The TOIB study. Health Technology Assessment (Winchester, England) 12(22): 4-155.

13. Al-Omari B (2019) Osteoarthritis and management. Ortho Res Online J 5(5): OPROJ.000622.

14. Landmark AMD, Ofstad EH, Svennevig J (2017) Eliciting patient preferences in shared decision-making (SDM): Comparing conversation analysis and SDM measurements. Patient Educ Couns 100(11): 20812087.

15. Elkin EB, Kim SH, Casper ES, Kissane DW, Schrag D (2007) Desire for information and involvement in treatment decisions: elderly cancer patients' preferences and their physicians' perceptions. J Clin Oncol 25: 5275-5280.

16. Politi MC, Dizon DS, Frosch DL, Kuzemchak MD, Stiggelbout AM (2013) Importance of clarifying patients' desired role in shared decision making to match their level of engagement with their preferences. BMJ 347: 7066 .

17. Al-Omari B, Sim J, Croft P, Frisher M (2015) Patient preferences for the pharmacological treatment of osteoarthritis: a feasibility study using adaptive choice-based conjoint analysis (ACBCA). European Journal for Person Centered Healthcare 3(2): 253-260. 
18. Friedrichs A, Spies M, Härter M, Buchholz A (2016) Patient preferences and shared decision making in the treatment of substance use disorders: A systematic review of the literature. PLoS One 11(1): e0145817.

19. Coulter A, Collins A (2011) Making shared decision-making a reality.

20. DoH (Department of Health) (2012) Liberating the NHS: No decision about me without me-Government response.

21. Weston W (2001) Informed and shared decision-making: the crux of patient-centered care. CMAJ 165(4): 438-439.

22. Montori VM, Gafni A, Charles C (2006) A shared treatment decisionmaking approach between patients with chronic conditions and their clinicians: The case of diabetes. Heal Expect 9(1): 25-36.

23. Legare F, Witteman HO (2013) Shared decision making: Examining key elements and barriers to adoption into routine clinical practice. Health Aff 32(2): 276-284

24. Carnes D, Anwer Y, Underwood M, Harding G, Parsons S, et al. (2008) Influences on older people's decision making regarding choice of topical or oral NSAIDs for knee pain: qualitative study. BMJ 336(7636): 142145.

25. Frewer LJ, Salter B, Lambert N (2001) Understanding patients' preferences for treatment: the need for innovative methodologies. Qual Health Care 10: 50-54.

26. Laba TL, Brien JA, Fransen M, Jan S (2013) Patient preferences for adherence to treatment for osteoarthritis: the M Edication Decisions in Osteoarthritis Study (MEDOS). BMC Musculoskeletal Disorders 14: 160.

27. Say RE, Thomson R (2003) The importance of patient preferences in treatment decisions -challenges for doctors. BMJ 327(7414): 542-545.

28. Brom L, Pasman HRW, Widdershoven GAM, van der Vorst MJDL, Reijneveld JC, et al. (2014) Patients' preferences for participation in treatment decision-making at the end of life: Qualitative interviews with advance cabcer patients. PLos ONE 9(6): e100435.

29. Al-Omari B (2017) Patient preferences for the pharmacological treatment of osteoarthritis using adaptive choice-based conjoint (ACBC) analysis: A pilot study. European Journal for Person Centered Healthcare 5(2): 220-224.

30. Ryan M, Scott DA, Reeves C, Bate A, van Teijlingen ER, et al. (2001) Eliciting public preferences for healthcare: a systematic review of techniques. Health Technol Assess 5(5): 1-186.

31. Hauber AB, Arden NK, Mohamed AF, Johnson FR, Peloso PM, et al. (2013) A discrete-choice experiment of United Kingdom patients' willingness to risk adverse events for improved function and pain control in osteoarthritis. Osteoarthritis and cartilage 21(2): 289-297.

32. Caruso E, Rahnev D, Banaji M (2009) Using conjoint analysis to detect discrimination: Revealing covert preferences from overt choices. Social Cognition 27(1): 128-137.

33. Bridges JFP, Onukwugha E, Johnson FR, Hauber AB (2007) Patient preference methods - A patient centered evaluation paradigm. ISPOR Connections 13(6): 4-7.

34. Chan F, Da Silva Cardoso E, Lam Illinois C, Miller SM (2004) Rehabilitation counseling students' attitudes toward people with disabilities in three social contexts: A conjoint analysis. Rehabilitation Counseling Bulletin 47(4): 194-204.

35. Lamiraud K, Geoffard PY (2007) Therapeutic non-adherence: a rational behaviour revealing patient preferences? Health Econ 16: 1185-1204.

36. Cunningham CE, Deal K, Chen Y (2010) Adaptive choice-based conjoint analysis: a new patient-centered approach to the assessment of health service preferences. The Patient 3(4): 257-273. 